\title{
Resonant Synchronization in Heterogeneous Networks of Inhibitory Neurons
}

\author{
Reinoud Maex and Erik De Schutter \\ Laboratory of Theoretical Neurobiology, Born-Bunge Foundation, University of Antwerp, B-2610 Antwerp, Belgium
}

\begin{abstract}
Brain rhythms arise through the synchronization of neurons and their entrainment in a regular firing pattern. In this process, networks of reciprocally connected inhibitory neurons are often involved, but what mechanism determines the oscillation frequency is not completely understood. Analytical studies predict that the emerging frequency band is primarily constrained by the decay rate of the unitary IPSC. We observed a new phenomenon of resonant synchronization in computer-simulated networks of inhibitory neurons in which the synaptic current has a delayed onset, reflecting finite spike propagation and synaptic transmission times. At the resonant level of network excitation, all neurons fire synchronously and rhythmically with a period approximately four times the mean delay of the onset of the inhibitory synaptic current. The amplitude and decay time constant of the synaptic current have relatively minor effects on the emerging frequency band. By varying the axonal delay of the inhibitory connections, networks with a realistic synaptic kinetics can be tuned to frequencies from 40 to $>200 \mathrm{~Hz}$. This resonance phenomenon arises in heterogeneous networks with, on average, as few as five connections per neuron. We conclude that the delay of the synaptic current is the primary parameter controlling the oscillation frequency of inhibitory networks and propose that delay-induced synchronization is a mechanism for fast brain rhythms that depend on intact inhibitory synaptic transmission.
\end{abstract}

Key words: computer; delayed response; inhibition; network; oscillator; rhythm

\section{Introduction}

Networks of inhibitory neurons with reciprocal synaptic connections are found from invertebrate retina (Hartline and Ratliff, 1972) up to mammalian hippocampus (Cobb et al., 1997), striatum, thalamus, cerebellum, and neocortex (Kisvarday et al., 1993; Tamás et al., 1998; Galarreta and Hestrin, 1999; Gibson et al., 1999; McBain and Fisahn, 2001). In many of these brain regions, fast-oscillating local field potentials (from 40 to $>200 \mathrm{~Hz}$ ) were recorded with subclasses of inhibitory neurons firing phaselocked to, and often thought to generate, the rhythm (Bragin et al., 1995; Whittington et al., 1995; Kandel and Buzsáki, 1997; Csicsvari et al., 1999, 2003; Grenier et al., 2001). The rhythms are typically confined within a frequency band characteristic of the brain area, the experimental procedure, or the associated behavior (for review, see Gray, 1994; Buzsáki and Chrobak, 1995; Baçar, 1998; Farmer, 1998; Traub et al., 1999).

If networks of inhibitory neurons are responsible for maintaining the rhythms, then the constancy in frequency in each particular case requires either that the individual neurons have an intrinsic resonance around the oscillation frequency or that populations of inhibitory neurons preferentially synchronize within the recorded frequency band.

\footnotetext{
Received July 19, 2003; revised Sept. 24, 2003; accepted Sept. 25, 2003.

This work was supported by Inter-University Attraction Pole Programme Grant 5104, Fonds voor Wetenschappelijk Onderzoek Grant G.0401.00 (Vlaanderen), and European Union Grant QLRT-2001-01241. We thank Mike Wijnants for graphics expertise and anonymous reviewers for contributions, particularly to Appendix.

Correspondence should be addressed to Reinoud Maex, Born-Bunge Foundation, University of Antwerp, Universiteitsplein 1, B-2610 Antwerp, Belgium. E-mail: reinoud@bbf.uia.ac.be.

Copyright $\odot 2003$ Society for Neuroscience $\quad$ 0270-6474/03/2310503-12\$15.00/0
}

Theoretical studies demonstrated that a homogeneous pair of reciprocally connected inhibitory neurons lacking intrinsic resonance can synchronize their spikes with zero phase lag, provided a finite delay separates the generation of an action potential by one of the neurons and the peak of the synaptic response, or IPSC, in the paired neuron (van Vreeswijk et al., 1994; Ernst et al., 1995). Most studies of inhibitory networks, analyzing the parameter dependency of synchrony, implemented the required delay as a slow rise time of the IPSC, thereby avoiding the analytical difficulties imposed by the discrete delays of axonal conduction and synaptic transmission. The frequency selectivity of the rhythms, however, is not completely understood and was attributed to the decay time constant of the synaptic response (Wang and Rinzel, 1993; Traub et al., 1996; Wang and Buzsáki, 1996).

Here we demonstrate that the discrete delay in the onset of the IPSC, which is attributable to the finite duration of axonal conduction and synaptic transmission, induces a resonance phenomenon in inhibitory networks, and the oscillation period at resonance is close to four times the combined axonal and synaptic delay. A delayed IPSC onset also enables synchrony to develop in networks with a fast-rising IPSC waveform, in accordance with recent experimental findings (Bartos et al., 2001, 2002; Carter and Regehr, 2002).

The present study elaborates on an inhibitory network model developed for reproducing $160 \mathrm{~Hz}$ oscillations recorded in the cerebellar cortex of transgenic mice deficient for calretinin and calbindin (Cheron et al., 2001; Maex et al., 2002). The observed frequency constancy made us search for a mechanism capable of inducing resonance in spatially organized, heterogeneous inhibitory networks. 


\section{Materials and Methods}

Model neurons. Fast-spiking model neurons had a spherical soma and an unbranched dendrite of five cylindrical compartments. All compartments had the same surface area and a passive membrane time constant of $30 \mathrm{msec}$. The spike-generating inactivating $\mathrm{Na}^{+}$channel and the delayed rectifier were restricted to the soma. All compartments had a highvoltage-activated $\mathrm{Ca}^{2+}$ channel, A-type and combined voltage- and $\mathrm{Ca}^{2+}$-activated $\mathrm{K}^{+}$channels, and a weak anomalous inward rectifier. Rate constants were converted to $37^{\circ} \mathrm{C}$, using $Q_{10}=3$ for voltage-gated and $Q_{10}=2$ for ligand-gated channels (Maex and De Schutter, 1998a). The reversal potential of the leak current was drawn from a uniform distribution between -70 and $-60 \mathrm{mV}$. The neurons did not exhibit subthreshold oscillations or intrinsic membrane resonance, nor did they fire postinhibitory rebound spikes. Suprathreshold current injection evoked narrow spikes $(0.3 \mathrm{msec}$ width at $-40 \mathrm{mV})$ without firing rate adaptation (Maex and De Schutter, 1998a, their Fig. 2 A). We selected this neuron model, which combines the active properties of a model cerebellar granule cell and the passive properties of a Golgi cell, because its firing rate increased linearly with the intensity of applied current over almost the entire dynamic range (from $15 \mathrm{~Hz}$ at the brisk $65 \mathrm{pA}$ threshold to $1000 \mathrm{~Hz}$, the maximum firing rate imposed by a $1 \mathrm{msec}$ absolute refractory period; mean slope, $0.65 \mathrm{~Hz} / \mathrm{pA}$ for an input resistance of $162 \mathrm{M} \Omega$ ).

Model $G A B A_{A}$ receptor synapses. A GABA receptor channel with a reversal potential of $-70 \mathrm{mV}$ was inserted on the somatic compartment. Each afferent action potential triggered a unitary conductance increase with a fixed, dual-exponential time course:

$g(t)=(w G A / n)\left(\exp (-(t-d) / \tau)-\exp \left(-(t-d) / \tau_{\mathrm{r}}\right)\right)$ for $t$

$$
>d \text { (and zero otherwise). }
$$

The parameters $w, \tau$, and $d$ are the relative peak conductance, decay time constant and delay of the synaptic response, respectively (see Fig. 2A), and $t$ denotes time elapsed after the rising phase of the afferent action potential crossed the $-20 \mathrm{mV}$ level. The parameter $d$ encompasses both axonal conduction and synaptic transmission delays. The normalization constants $A$ and $n$ (the number of afferent synapses) ensured that the summed unitary conductances had a peak amplitude equal to $w G$ when $\tau$ or $n$ was varied. The dimensionless parameter $w$ is used only for reference. For $w=1$, the summed peak conductance $w G$ was $3 \mathrm{mS} / \mathrm{cm}^{2}$. The rise time constant $\tau_{\mathrm{r}}$ determines the shape of the conductance response and was taken as $\tau / 27.4$ to reproduce the IPSC waveform of hippocampal pyramidal neurons (Ropert et al., 1990). For $\tau=3 \mathrm{msec}, \tau_{\mathrm{r}}=0.11 \mathrm{msec}$, which is comparable after temperature correction to the mean rise time constant of $0.16 \mathrm{msec}$, recorded in basket cells of dentate gyrus at $34^{\circ} \mathrm{C}$ (Bartos et al., 2002). In selected simulations (see Fig. 7B), neurons reciprocally connected through $\mathrm{GABA}_{\mathrm{A}}$ receptor synapses also made electrical synapses on the same compartments (Tamás et al., 2000). These gap junctions were pure resistors with a conductance expressed as a fraction of the peak conductance of the associated chemical synapse.

Conversion to physiological data. Because conductances are expressed as densities over the compartments to which the channels are attached, the output of this neuron model is primarily invariant over spatial scaling. For a neuron with total membrane area $S$, the unitary peak conductance $g_{\text {unitary }}$ can be derived as:

$$
g_{\text {unitary }}=\frac{w G S}{6 n} .
$$

The factor 6 in the denominator converts density over the somatic compartment to density over the entire model neuron. Hence, taking $w G=3$ $\mathrm{mS} / \mathrm{cm}^{2}$, the afferent synapses of a neuron with total area $S=12,000$ $\mu \mathrm{m}^{2}$ (Bartos et al., 2001) and $n=60$ (Wang and Buzsáki, 1996) have a unitary peak conductance of $1 \mathrm{nS}$.

Afferent fibers. The dendritic compartments received AMPA receptor synapses (rise and decay time constants, 0.03 and $0.5 \mathrm{msec}$, respectively; summed peak conductance, $G=16.1 \mathrm{mS} / \mathrm{cm}^{2}$; Maex and De Schutter, 1998a) from a population of afferent fibers. These fibers were Poisson processes, all firing at the same constant rate throughout a simulation. The time histogram of spike counts of the fiber population had a flat power spectrum. Because synapses and fibers are computationally expensive, a diluted population of fibers was simulated. Each fiber represented the compound spike trains from several tens of excitatory fibers and fired at a rate varied between simulations from 50 to 12,800 spikes/sec.

Model network configurations. We first examined how the parameters of the inhibitory synapse affect the oscillation frequency and power in a network characterized by single values for $d, \tau$, and $w$. We then proceeded to validate the derived relationships on networks with distributed delays and weights and with stochastic connections.

In the one-dimensional network with only nearest neighbor coupling, 100 model neurons were positioned on a one-dimensional array. The neurons made autapses, with zero delay, and synapses on the closest neighbor on each side with delay $d$. This overtly simplified network has the advantage of being completely characterized by the single delay value $d$, the single decay time constant $\tau$, and the single synaptic weight value $w$. We varied systematically the values of $d, \tau$, and $w$ (see Figs. 1, 2, 3A, 7).

In the one-dimensional network with distributed delays, each neuron was connected to all neighbors within an axon or connection radius $r$ (measured in units of interneuron distance) with the delays $d$ set proportional to the interneuron distances. Assuming a spacing of $300 \mu \mathrm{m}$ between the neurons and an axonal conduction speed of $0.3 \mathrm{~m} / \mathrm{sec}$, the delay to the nearest neighbor measured $1 \mathrm{msec}$, and delays to more distant neurons were an integer multiple of this. The mean delay of the network connections measured $(r+1) / 2$. We varied systematically the values of $r$ and $\tau$ (Figs. 3B, 4).

The two-dimensional network, representing circuits with a predominantly radial connection pattern, was an array of $30 \times 30$ neurons. Their somata were positioned on a triagonal grid so that each neuron had six equidistant neighbors. Connecting a neuron to all neighbors alike within a radius $r$ makes the delay distribution very skewed and biased toward the largest delay values. Either the connection probability or the synaptic weight, therefore, needed to fall off with distance from the source neuron. In the network documented in Figure 5, synaptic weight decreased exponentially with a space constant twice the interneuron distance. Using $r=8$ and a constant connection probability of 0.6 , each neuron connected on average to 115 neighbors. The mean delay of the network was the mean $d$ over all connections, each $d$ weighted by the corresponding synaptic weight $w$ and with the exclusion of autapses, which did not contribute to synchrony. We used $\tau=3 \mathrm{msec}$.

For the effects of network size and connectivity (Fig. 6), nontopographic networks were simulated in which all neurons were stochastically connected with a variable probability. All synaptic connections had the same fixed delay, $d=1 \mathrm{msec}$ and $\tau=3 \mathrm{msec}$.

A population of afferent fibers was evenly distributed over the entire network space. The fibers had a conduction speed of $0.3 \mathrm{~m} / \mathrm{sec}$ and radiated $2.5 \mathrm{~mm}$ in a plane perpendicular to the dendritic shafts, making en passant excitatory synapses on the dendritic compartments with a probability of 0.5 . There were 8100 fibers in the two-dimensional network and 4000 fibers in the one-dimensional and nontopographic networks (except when stated otherwise), providing each neuron on average with 424 and 320 synapses, respectively.

Boundary conditions. As in our previous studies, normalizing the unitary synaptic conductance over the number of afferent synapses prevented the possibility that the boundary neurons would fire at rates different from the network average. This normalization is justified because it is shown that our main findings are robust to great variability among the neurons in the number of synapses (Fig. 6A). In addition to having fewer synapses, with consequently stronger weights, neurons positioned close to the boundaries had a selective deficit of long connections. The resulting decrease in the mean synaptic delay, which is largest for a neuron positioned at a distance half the connection radius from the boundary, was relatively small. For example, in the one-dimensional network with $r=16$, the mean delay of the inhibitory synapses onto neurons 9 and 92 was 7.2 instead of $8.5 \mathrm{msec}$. Although these inhomogeneities at the boundaries did have a desynchronizing effect on the entire network, the value of the resonance frequency was hardly affected $(42.65 \mathrm{~Hz}$ in a 100 -neuron array, $42.18 \mathrm{~Hz}$ for the central 100-neuron segment of a 200-neuron array, and $42.57 \mathrm{~Hz}$ in a 100 -neuron ring). 
Noise and heterogeneity. The networks were noisy and heterogeneous, owing to the randomness of excitation and the randomization of the resting membrane potentials (see above) and synaptic weights (Maex and De Schutter, 1998a). This heterogeneity made the neurons in a disconnected network fire irregularly and with slightly different rates [coefficient of variation $(\mathrm{CV})$ of their firing rate, averaged over all excitation levels, 0.046; CV of their interspike intervals (ISIs), averaged over all neurons, 0.155$]$. These values typically increased in connected networks because lateral inhibition tended to amplify differences in the firing rate ( $\mathrm{CV}$ of the firing rate in the one-dimensional network with only nearest neighbor coupling, 0.072; CV of ISIs, 0.210). Finally, a simulation run started from random membrane potentials distributed uniformly between -90 and $-20 \mathrm{mV}$.

Implementation and simulation. Neuronal activity was calculated numerically with a modified version of the Genesis simulator (http://www. genesis-sim.org/GENESIS), using Crank-Nicolson integration in 20 $\mu$ sec steps. We used a random-number generator with two seed variables to avoid correlations in firing among the afferent fibers.

Analysis of network activity. We monitored network activity as the time series of spike counts in bins of 0.5 msec width (Fig. $1 A, D$ ). The power spectrum or periodogram was estimated, using the fast Fourier transform algorithm, as the average of 256-point overlapping Hann (cosine bell) windows (Press et al., 1988). In each window, the mean number of spikes was calculated first and subtracted from the individual counts (or, equivalently, power at $f=0$ was set at 0 ) so that the total power almost equaled the variance of the time signal. Coherent oscillations were quantified by the peak power and the associated center or network frequency. Network frequency was assessed at higher resolution (512 or 1024 point windows) for networks with a low resonance frequency.

To derive the resonance frequency of a network, the network was excited to various levels by incrementing the mean firing rate of the afferent fibers by a factor of the square root of 2 at each new run. The obtained average firing rates of the neurons encompassed their entire dynamic range ( 10 to $>900 \mathrm{spikes} / \mathrm{sec}$ ). A simulation run produced at least 1000 spikes for each neuron (500 in two-dimensional networks) and had a minimum duration of $3 \mathrm{sec}$. Over such long runs, power spectra were well reproducible and independent of initial values. From the power spectra obtained at different levels of excitation, a tuning curve was constructed after application of the following normalization procedure. We first noticed that a disconnected network produced a power spectrum with a single peak that was located at the mean neuronal firing rate. This peak had a height almost proportional to the firing rate. In accordance with this, the variance of the time series of spike counts scaled almost linearly with the mean spike count. This relationship between power and firing rate is compatible with a Poisson distribution of spike counts in the bins of the time signal. We therefore divided the peak power obtained at each level of excitation by the associated mean neuronal firing rate, correcting in this way for the power already present in the disconnected network. Finally, peak power scales in a synchronous network quadratically with the number of neurons $N$ and was therefore normalized to a network of $N=100$. All frequency-tuning curves plot peak power, divided by the mean neuronal firing rate and by $(N / 100)^{2}$ and multiplied by a scaling factor 1000 .

Resonance frequency $\left(f_{\mathrm{R}}\right)$ is the mean frequency of the tuning curve, which was constructed from at least eight levels of excitation. Taking this weighted average reduces errors attributable to sampling of the tuning curve. If the central peak of the curve was positioned very asymmetrically, the low-power tail was cut to avoid a systematic overestimation and underestimation of $f_{\mathrm{R}}$ in networks with low and high $f_{\mathrm{R}}$, respectively. Some tuning curves had a satellite peak at lower frequencies, and $f_{\mathrm{R}}$ may have been slightly underestimated in these networks (e.g., see Fig. $6 B$ ). Overall, errors on $f_{\mathrm{R}}$ are judged to be small because of the systematic attraction of network frequency toward $f_{\mathrm{R}}$ (see Fig. $5 B$ ).

Peak power is a combined metric of synchrony and rhythmicity and quantifies the network oscillations in a manner comparable with the analysis of experimentally recorded local-field potentials. A metric frequently used in the computational literature is the coherence index (Wang and Buzsáki, 1996; Bartos et al., 2001, 2002) (variations in White et al., 1998; Tiesinga et al., 2000). The coherence index (CI), taking values between 0 and 1, measures exclusively synchrony and assesses, averaged over all neuron pairs of the network, the fraction of coincident spikes. The probability of coincidence increases with the bin width of the discretized spike trains, which by convention is taken in $1 / 10$ of the oscillation period. Measuring only the $\mathrm{CI}$ is justified in the above studies because the neurons being excited by constant-current injection were almost perfect oscillators, and the inhibitory synapses constituted the only synchronizing mechanism. In the present study, in contrast, excitation is provided by afferent fibers, which carry random spike trains that can contribute to the synchronization of common efferent neurons (Maex et al., 2000). We mention for each spike raster plot the CI in the figure legend.

\section{Results}

We investigated the critical parameters for synchronization and frequency control in a heterogeneous network of model inhibitory neurons, themselves lacking intrinsic resonance. Randomly firing afferent fibers excited the neurons by continually activating AMPA receptor synapses on the dendrites. This noisy but realistic way of driving the neurons accentuated network-induced rhythmicity. Each neuron inhibited its neighbors, within a varying axon radius, through $\mathrm{GABA}_{\mathrm{A}}$ receptor synapses located on the somatic compartment. The delay $d$, strength $w$, and decay time constant $\tau$ of the IPSC (see Fig. $2 A$ ) were varied, and their effects on network dynamics were compared at various levels of network excitation.

\section{High-frequency oscillations emerge in networks with axonal and synaptic delays}

For a better understanding of the resonance mechanism, we first illustrate the results of a one-dimensional array in which a neuron is connected on each side only to the closest neighbor.

Figure $1 B$ demonstrates waxing and waning high-frequency oscillations in a network in which the combined synaptic and conduction delay $d$ was set to $1 \mathrm{msec}$, which is a typical latency value for inhibitory synaptic currents evoked from nearby neurons in paired recordings (Bartos et al., 2001). As a control, no oscillations were observed in the same network if the onset of the IPSC was instantaneous (delay $d=0$; Fig. $1 A, C$ ). This latter finding is in accordance with analytical studies proving that synchronous firing is unstable if the rise time of the synaptic response is shorter than the duration of the afferent spike (van Vreeswijk et al., 1994). However, using a synaptic response function with slower rise time compared with this control, e.g., an $\alpha$-function with instantaneous onset and peak conductance at $1 \mathrm{msec}$, did not improve synchrony in this sparsely connected network without axonal and synaptic delays.

The oscillation frequency and power were measured at various levels of network excitation spanning the entire dynamic range of the neurons. Figure $1 F$ shows that the tuning curve of the delayed network was centered at a resonance frequency $f_{\mathrm{R}}$ of 210 $\mathrm{Hz}$, with power falling off steeply at lower and higher levels of excitation. In the network without delays, no robust oscillations could be evoked at any frequency (Fig. $1 E$ ).

At the resonant level of network excitation, the mean firing rate of the neurons was close to the oscillation frequency of the network because each neuron fired a single spike at almost every cycle (Fig. 1D). At nonoptimal levels of excitation, the neurons changed their firing pattern to maintain a network frequency deviating from their firing rate but closer to $f_{\mathrm{R}}$. In particular, at low excitation levels, neurons skipped cycles of the oscillation. As a consequence, adjacent neurons could fire in alternate order (antiphase synchrony) at a rate half the network frequency (Fig. $1 F$, open data points). At high excitation levels, neurons fired multiple spikes at each single cycle, without affecting much the 


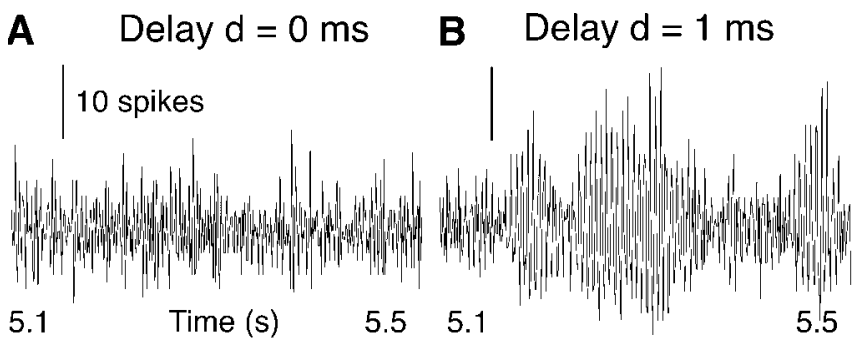

5.1

$5.5 \quad 5.1$
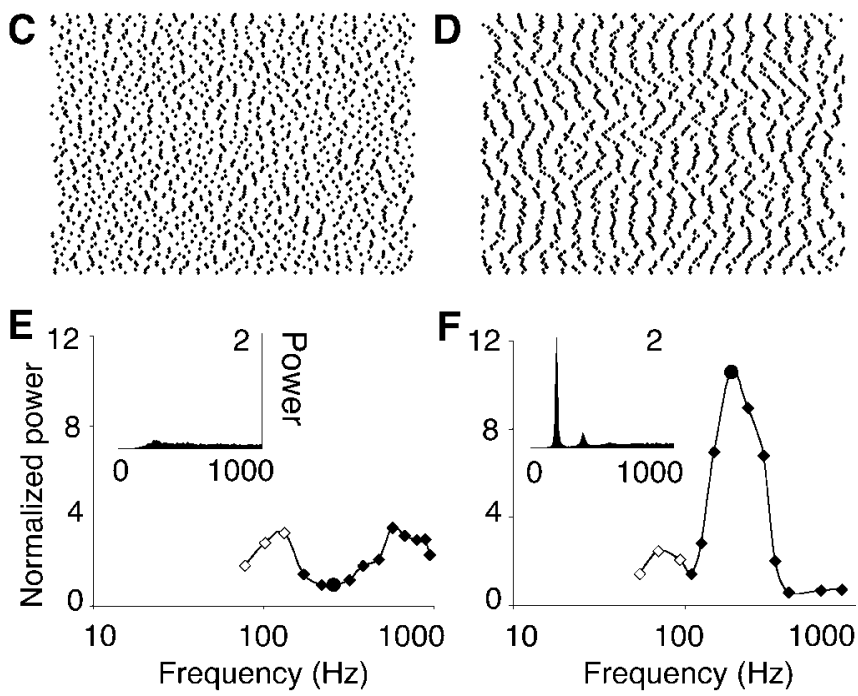

Figure 1. Resonance in a delayed inhibitory network. The activity of a one-dimensional, nearest neighbor-coupled network of 100 neurons is illustrated for two delay values of the synaptic response: $d=0 \mathrm{msec}(A, C, E)$ and $d=1 \mathrm{msec}(B, D, F)$. The synaptic decay time constant $\tau$ was 3 msec. $A, B$, Time histograms of the number of spikes generated by the entire population of neurons (bin width, $0.5 \mathrm{msec}$ ). Horizontal bars indicates zero level. C, D, Raster plots of spikes fired during the time interval indicated by the horizontal bars in $A$ and $B$. Each dot represents a spike ( $x$-axis, time; $y$-axis, position of the neuron along the array). In the network with $d=1 \mathrm{msec}(D ; \mathrm{Cl}=0.110)$, most neurons fired at each oscillation cycle; hence, the network frequency ( $187 \mathrm{~Hz})$ was close to the mean neuronal firing rate ( $181 \pm 10$ spikes $/ \mathrm{sec})$. In the network with zero delay $(C ; \mathrm{Cl}=0.087)$, spikes were produced at a similar rate $(172 \pm 16$ spikes/sec), but their spatiotemporal patterning was lost. $E$, $F$, Insets show periodograms [units $\left.1(0.5 \mathrm{msec})^{2}\right]$ of the spike count histograms shown in $A$ and $B$ averaged over $7 \mathrm{sec}$. Tuning curves plot peak power and network frequency obtained at various levels of network excitation (filled circle in each tuning curve: data from $A, B$ ). In the simulations represented by open points, adjacent neurons fired in antisynchrony (van Vreeswijk et al., 1994); hence, network frequency was twice the mean neuronal firing frequency.

cycle duration. This doublet or burst firing was most prominent in networks with delays larger than the present $1 \mathrm{msec}$ value (data not shown).

\section{The delay parameter determines the resonance frequency}

Several studies of inhibitory networks demonstrated that the oscillation frequency decreases when the strength, decay time constant, or delay of the inhibitory synaptic response are increased (Bush and Sejnowski, 1996; Traub et al., 1996; Wang and Buzsáki, 1996; Pauluis et al., 1999; Bartos et al., 2002; Liley et al., 2002). Figure 2 illustrates the complementary effects of these parameters, i.e., the effects on the frequency-tuning curve of a network.

The frequency tuning was almost invariant over the value $w$ of the synaptic strength (Fig. $2 \mathrm{~B}$ ). In the network with a resonance frequency $f_{\mathrm{R}}$ of $210 \mathrm{~Hz}$, illustrated above, a variation in peak conductance by a factor of 8 suppressed the mean firing rate on average by $51 \%$, but $f_{\mathrm{R}}$ decreased only $14 \%$ (from 225 to $183 \mathrm{~Hz}$ ).
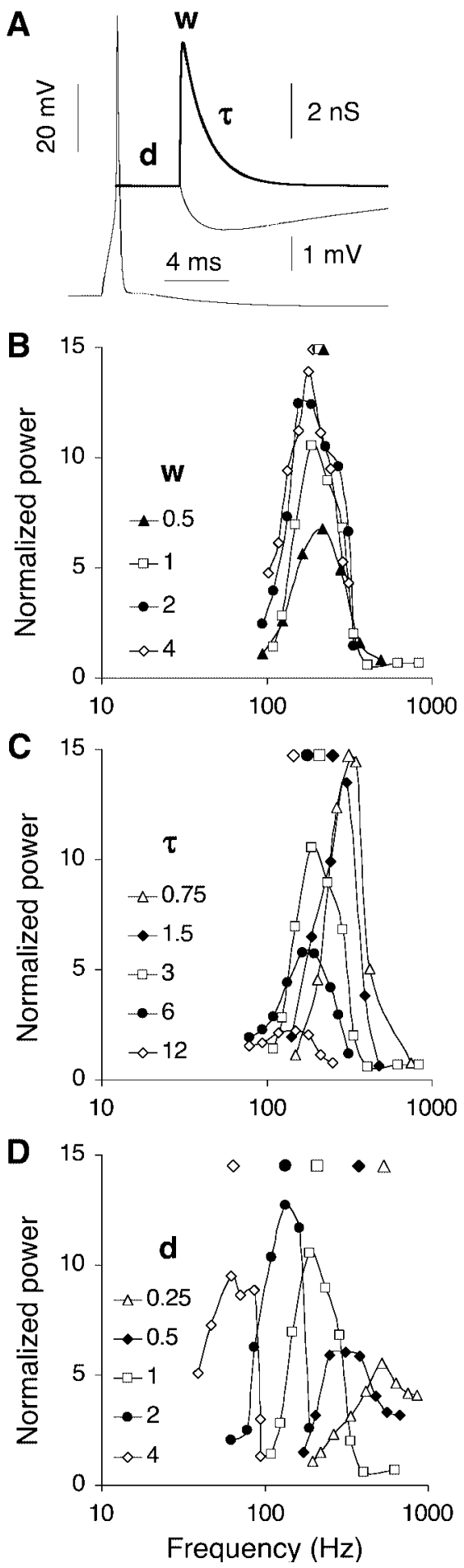

Figure 2. Dependence of resonance frequency on the parameters of the inhibitory synaptic response. $A$, The unitary inhibitory synaptic response, which is a transient conductance increase of the $\mathrm{GABA}_{A}$ receptor channel (bold curve), follows an action potential of the presynaptic neuron after a delay $d$, reaches (relative) peak conductance $w$, and decays exponentially with time constant $\tau$. The middle trace is the (vertically offset) somatic postsynaptic potential. $B-D$, Tuning curves of a network with only nearest neighbor coupling for various values of $w, \tau$, and $d$. For each parameter set, the resonance frequency is indicated by the corresponding symbol at the top. $B$, Tuning curves for four values of $w(d=1 \mathrm{msec} ; \tau=3 \mathrm{msec} ; w=1$ corresponds to a synaptic peak conductance density $\left.G=3 \mathrm{mS} / \mathrm{cm}^{2}\right)$. C, Tuning curves for five values of $\tau$, indicated in milliseconds ( $d=1 \mathrm{msec} ; w=1$, except for $\tau=12 \mathrm{msec}$, for which $w=0.5)$. D, Tuning curves for five values of $d$ indicated in milliseconds ( $w=1 ; \tau=3 \mathrm{msec}$, except for $d=$ $4 \mathrm{msec}$, for which $\tau=6 \mathrm{msec}$ ). 


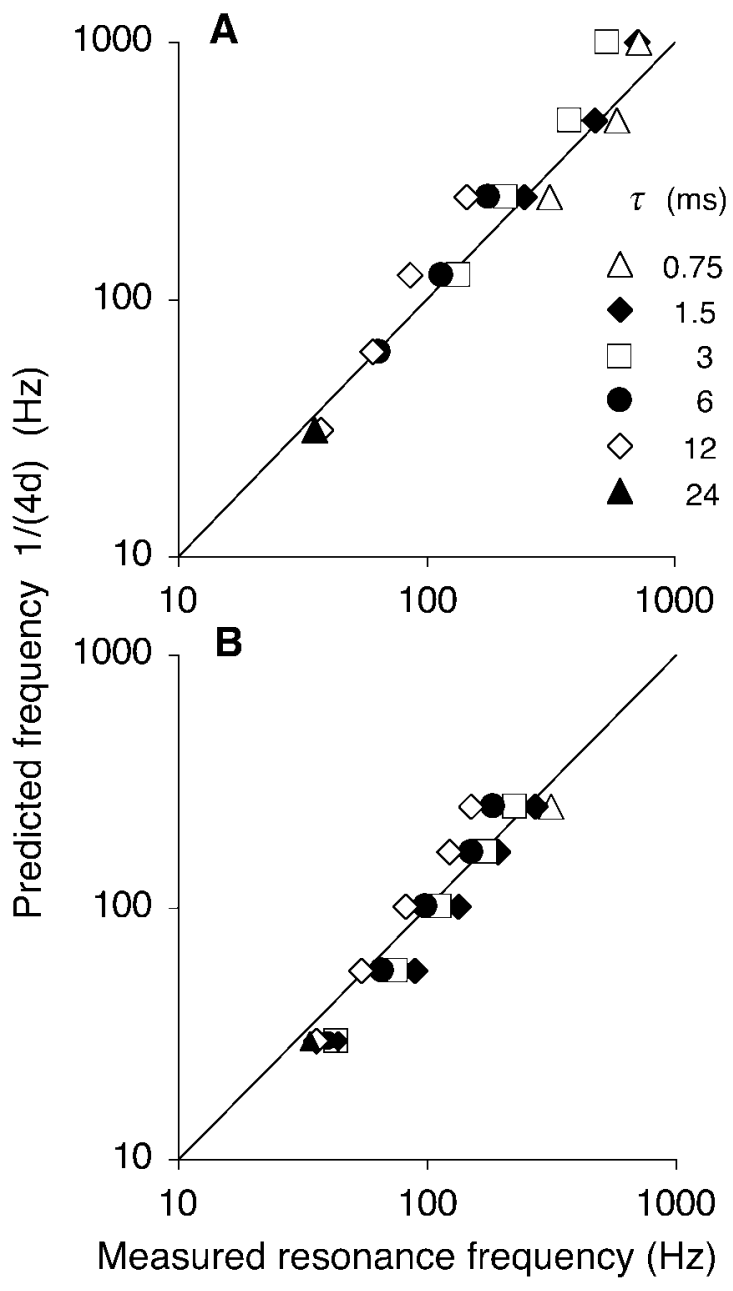

Figure 3. Predicted versus measured resonance frequency. The resonance frequency $f_{R}$ derived from the tuning curves ( $x$-axis) is compared with $1 /(4 d)$ ( $y$-axis). The diagonal lines are the identity curves, indicating perfect prediction. $A$, Network with only nearest neighbor coupling for various delays $d$ of the synaptic response $(0.25-16 \mathrm{msec})$. Symbols represent different values of the decay time constant $\tau$, indicated in milliseconds. For each $d, \tau$ pair, the network with weight $w$ producing maximal power was selected. The effect of varying $w$, which is not illustrated, was always smaller than the effect of varying $\tau . B$, Network with $d=1 \mathrm{msec}$ from $A$ for increasing degrees of connectivity (axon connection radii $r, 1-16$ ) and hence for increasing values of the mean delay $d(1-8.5 \mathrm{msec})$. At the highest connectivity $(r=16)$, resonance was clustered between $34 \mathrm{~Hz}$ ( $\tau=24 \mathrm{msec}$ ) and $44 \mathrm{~Hz}(\tau=1.5 \mathrm{msec})$.

We disregard this weight parameter further and present the tuning curve with largest power for networks simulated at various weight values. Increasing or decreasing the exponential decay time constant $\tau$ of the postsynaptic current displaced the tuning curve to lower and higher frequencies, respectively (Fig. 2C). The resonance frequency $f_{\mathrm{R}}$ decreased from $338 \mathrm{~Hz}$ at $\tau=0.75 \mathrm{msec}$ to $149 \mathrm{~Hz}$ at $\tau=12 \mathrm{msec}$. This effect was small, however, compared with the inverse relationship obtained between $f_{\mathrm{R}}$ and the delay $d$ of the synaptic response (Fig. $2 D$ ). Varying $d$ from 0.25 to $4 \mathrm{msec}$ decreased $f_{\mathrm{R}}$ from 536 to $65 \mathrm{~Hz}$.

Over a broad range of values of the delay $d$ and the decay time constant $\tau, f_{\mathrm{R}}$ approximated $1 /(4 d)$ (Fig. $3 A$ ). At the lowest delay values $(0.25$ and $0.5 \mathrm{msec})$, the measured $f_{\mathrm{R}}$ was $<1 /(4 d)$ as the network frequency approached $1000 \mathrm{~Hz}$, the maximal neuronal firing rate. Networks with a (single) large delay value, on the other hand, could have multimodal tuning curves because at high excitation levels, the neurons started firing bursts, in particular when $\tau$ was much less than $4 d$ (the oscillation period at reso-
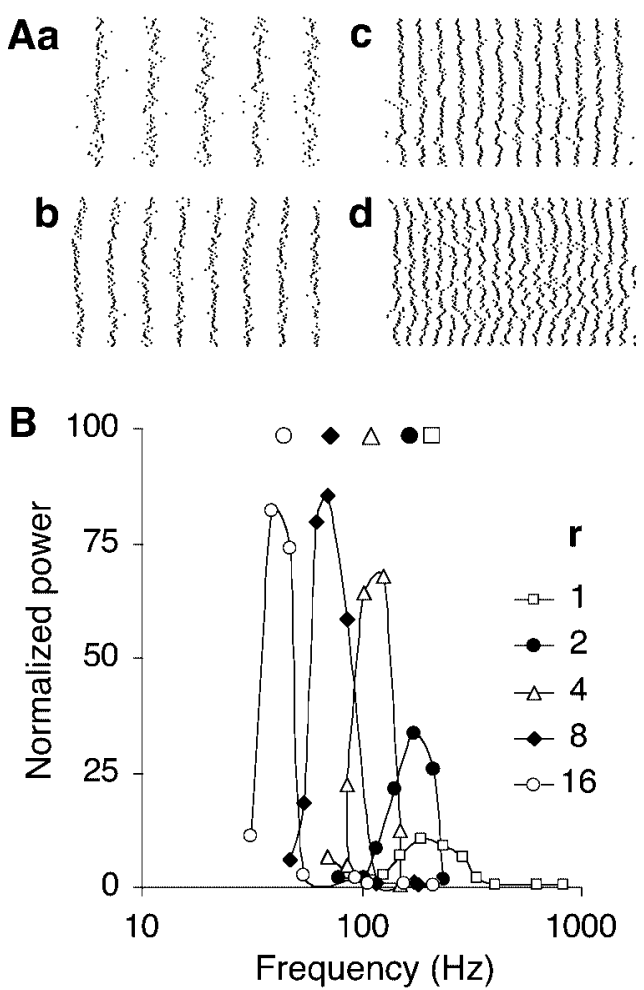

Figure 4. Inverse relationship between the connection radius $r$ of the one-dimensional network and the resonance frequency $f_{\mathrm{R}} . A$, Spike raster plots compared for various axonal connection radii $r$ (in units of interneuron distance; $\tau=3 \mathrm{msec} ; w=1$ ). The raster plots (width, 100 msec) were obtained at firing rates close to resonance for $r=16(a ; 47 \mathrm{~Hz}$ oscillations; $\mathrm{Cl}=$ $0.303), r=8(b ; 70 \mathrm{~Hz} ; \mathrm{Cl}=0.302), r=4(c ; 125 \mathrm{~Hz} ; \mathrm{Cl}=0.234)$ and $r=2(d ; 172 \mathrm{~Hz} ; \mathrm{Cl}=$ 0.153). The format is the same as in Figure 1D, which shows the raster plot for $r=1 . B$, Frequency-tuning curves of networks with the indicated connection radii $r$. Tuning curves for $r \leq 4$ were obtained with a 100-neuron array. The tuning curves for $r=8$ and 16 were calculated from the central 100-neuron segment of a 200-neuron array.

nance). Such unrealistic networks did not always have a clear optimal frequency, and their data were not included in Figure $3 \mathrm{~A}$ (but see next section).

A different value of $\tau$ was optimal for different values of $d$ (Fig. $3 A$ ), a nonoptimal $\tau$ driving $f_{\mathrm{R}}$ off the predicted frequency of $1 /(4 d)$ with a considerable loss of power (Fig. $2 C$ ). For example, oscillations of $\sim 250 \mathrm{~Hz}$ (obtained with $d=1 \mathrm{msec}$ ) were optimal with $\tau=0.75-3 \mathrm{msec}$. Oscillations of $\sim 62.5 \mathrm{~Hz}(d=4 \mathrm{msec})$ required a $\tau$ of $6-12 \mathrm{msec}$, a value comparable with the recorded $10 \mathrm{msec}$ decay of (probably compound) IPSCs during hippocampal gamma oscillations (Traub et al., 1996). With $\tau \leq 3 \mathrm{msec}$, networks with only nearest neighbor coupling were unable to generate oscillations $<100 \mathrm{~Hz}$.

\section{Distributed delays increase the robustness of frequency control}

Increasing the connection radius $r$ of the one-dimensional network, with each neuron now connected on each side to its $r$ nearest neighbors, improved the synchronization process as assessed from a rise in the CI from 0.11 to $>0.3$ (Fig. $4 A$ ). Networks with larger connection radii produced oscillations of greater amplitude and lower frequency (Fig. 4B). The period at resonance was equal to approximately four times the mean delay $d$ of the synaptic response (Fig. 3B).

Increasing the connection radius made the oscillations more robust at nonoptimal values of $\tau$. As each neuron was connected 
to neighbors positioned at various distances, within a fixed axon radius $r$ and with delays proportional to distance, a rectangular distribution of delays was formed. Inhibition through synapses with distributed delays made resonance less dependent on the appropriate value of $\tau$ because synchronously firing neurons at increasing distances activated their synapses with staggered delays. More particularly, in a linear array, the most distant afferent neuron would activate its synapse with a delay almost twice the mean delay, i.e., halfway in the oscillation cycle. This temporal summation of IPSCs induced a more lasting inhibition in the postsynaptic neuron, comparable with that induced by the activation of a single slowly decaying synapse. As a consequence, with a realistic decay time constant as small as $1.5-3 \mathrm{msec}$ (Bartos et al., 2001; Carter and Regehr, 2002), resonance could be induced in the entire gamma frequency range and beyond by varying only the size of the axonal connection radius $r$ (Figs. 3B, $4 B$ ).

The same relationship between mean delay and resonance was observed in twodimensional networks, provided the connection probability between two neurons, or the connection weight, tapered off with distance to obtain a uniform distribution of synaptic delays or weighted delays. For example, the tuning curve in Figure $5 \mathrm{~A}$ peaked at $78 \mathrm{~Hz}$, i.e., at a period four times the mean weighted delay of network connections (3.2 msec). The tuning was sharp, oscillations being produced only within a narrow frequency band around $f_{\mathrm{R}}$ for levels of excitation ranging from 12 to 375 spikes/sec, i.e., for all but the two rightmost excitation levels plotted in Figure 5B. Hence, although resonance was achieved when the neuronal firing rate equaled the oscillation frequency, oscillations at frequencies close to $f_{\mathrm{R}}$ were generated over a broad domain of firing rates, encompassing almost the entire dynamic range of the neurons. Indeed, levels of excitation that produced average firing rates from 22 to 913 spikes/sec in a disconnected network evoked oscillations in the connected network restricted to a $47-149 \mathrm{~Hz}$ band (Fig. $5 B$ ).

Figure 5, $C$ and $D$, illustrates in greater detail some observations already mentioned for the one-dimensional network. At resonance (Fig. 5C), the mean neuronal firing rate (73.4 spikes/ $\mathrm{sec}$ ) was close to the network frequency on the periodogram (78 $\mathrm{Hz}$; Fig. $5 C, a$ ). The neurons fired a single spike at each cycle, producing a single peak at $13 \mathrm{msec}$ on the averaged interspike interval histogram (Fig. $5 C, b$ ). The raster plot (Fig. $5 C, c$ ) and action potential trajectories (Fig. $5 C, d$ ) further demonstrate that synchrony was very robust though not very precise $(\mathrm{CI}=0.287)$. At the next lower level of excitation (Fig. 5D), network frequency $(72 \mathrm{~Hz}$; Fig. $5 D, a)$ was higher than the average firing rate (45 spikes/sec) and close to $f_{\mathrm{R}}(83 \mathrm{~Hz})$. The interspike interval histogram (Fig. 5D,b) showed a main peak at $25 \mathrm{msec}$, i.e., approximately twice the period at resonance. The synchrony induced at the onset of excitation rapidly faded away (Fig. $5 D, c$ ), but $72 \mathrm{~Hz}$

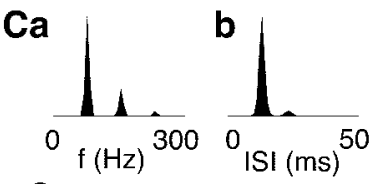

c
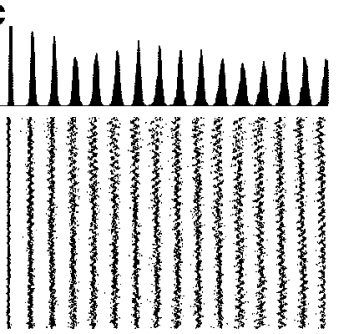

0

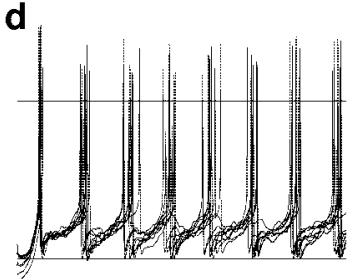

0

Time (s)

0.1 b

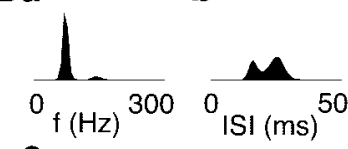

c.
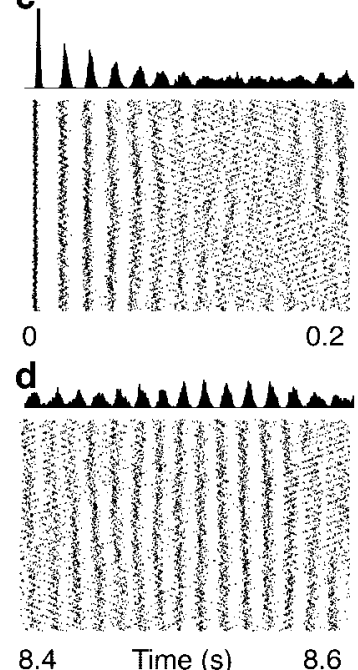

Figure 5. Resonance in a two-dimensional network. $A$, Tuning curve of a triagonal network of 900 neurons, with mean weighted delay $d=3.2 \mathrm{msec}$ (decay time constant $\tau=3 \mathrm{msec}$ ). $B$, Frequency range of the emerging network oscillations. Over the various levels of excitation, the mean neuronal firing rate varied from 12 to $598 \mathrm{spikes} / \mathrm{sec}(x$-axis). The resulting network

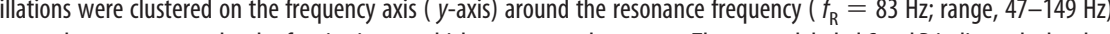
rex firing rate, $73.4 \mathrm{spikes} / \mathrm{sec}$ ). a, Periodogram of network activity over a simulation run of $8 \mathrm{sec}$ , Membrane potential traces of nine randomly selected neurons (the neurons with array indices 100, 200, . . 900) of the individual spikes fired between 8.2 and 8.4 sec after the start of the simulation $(\mathrm{Cl}=0.092$ or 0.147 after correction for the mismatch between firing rate and network frequency; Tiesinga and José, 2000).

oscillations kept waxing and waning throughout the simulation (Fig. 5D, d).

\section{Resonance arises in networks with sparse, asymmetrical connections}

To determine the critical synaptic number for resonance, the mean number of connections per neuron was varied, whereas total synaptic weight was kept constant, in a network without topographic ordering, i.e., with each neuron able to connect to each other neuron with a single delay $d=1 \mathrm{msec}$. A network with a mean number of five synapses per neuron produced at resonance a power close to that achieved in the fully connected network (Fig. 6A, open circles). This threshold did not depend on the connection rule because power hardly increased when the network was randomly connected using the restriction that all neurons received the same number of synapses (Fig. $6 \mathrm{~A}$, filled diamonds). This absolute threshold for synchronization was further independent of the size of the network and the summed synaptic weight, and it was always less than the number of synapses needed to obtain synchrony in a network without delays (Fig. 6A, dashed curve).

The tuning curves (Fig. $6 B$ ) of the sparsely connected network ( 5 synapses per neuron; open squares; $f_{\mathrm{R}}=225 \mathrm{~Hz}$ ) and the fully 

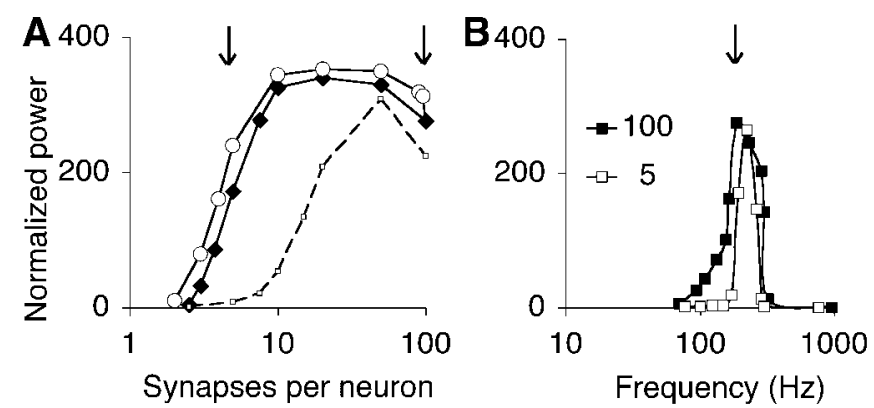

$\mathrm{Ca}$

Synapses per neuron

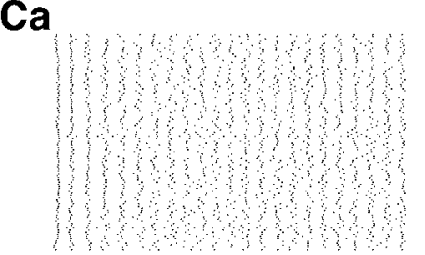

b

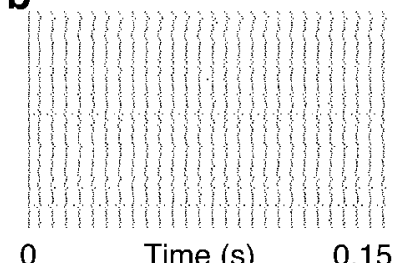

$\mathrm{Da}$

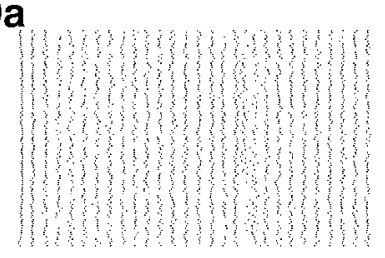

b

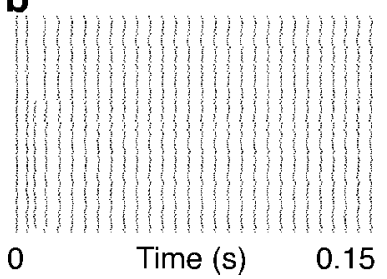

Figure 6. The threshold number of synapses for resonance is small. $A$, The power at approximately the resonant level of network excitation (see $B$, arrow) is compared for two stochastic connection rules in a network of 100 neurons with varying degrees of connectivity (open circles, filled diamonds; delay $d=1 \mathrm{msec}$; decay time constant $\tau=3 \mathrm{msec}$ ). Either each neuron was connected with a fixed probability to each other neuron, producing a binomial distribution of synaptic numbers (filled diamonds; the $x$-axis gives mean number of synapses per neuron), or, alternatively, for each neuron, a fixed number of afferents was randomly selected (open circles). In the control simulations (dashed curve), the synaptic response was modeled as an $\alpha$ function with zero delay and a time constant of $1 \mathrm{msec}$, producing a peak conductance at $1 \mathrm{msec}$ and a resonance frequency at $300 \mathrm{~Hz}$. $B$, Frequency-tuning curve of the sparsely connected network with, on average, five synapses per neuron (open squares; see $A$, left arrow) and of the fully connected network (filled squares; 100 synapses; see A, right arrow). C, Raster plots of spikes in the sparsely connected network (mean number, 5 synapses per neuron). Note that neurons 11 and 53 were not connected to by any other neurons using this stochastic connection rule. The neurons were excited either by afferent fibers $(a ; \mathrm{Cl}=0.178)$ or by the intrasomatic injection of the equivalent constant current $(b ; \mathrm{Cl}=0.381) . D$, Raster plots for the fully connected network excited by afferent fibers $(a ; \mathrm{Cl}=0.237)$ and current injection $(b ; \mathrm{Cl}=0.465)$.

connected network (100 synapses; filled squares; $f_{\mathrm{R}}=214 \mathrm{~Hz}$ ) were centered at approximately the same resonance frequency as the tuning curve of the network with only nearest neighbor coupling, shown in Figure $1 F\left(f_{\mathrm{R}}=210 \mathrm{~Hz}\right)$. The sparsely connected network was the more narrowly tuned (Fig. $6 B$ ) because antiphase synchronization produced a broader falloff at low frequencies in the fully connected network.

\section{Effects of noise and heterogeneity}

The randomization of synaptic weights and resting membrane potentials and the randomness in the activation of excitatory synapses made the present network heterogeneous and noisy.

We excited the nontopographic network in a noiseless manner by injecting a constant current into each neuron. The raster plots in Figure 6, $C$ and $D$, compare the firing patterns of the sparsely (Fig. 6C) and fully connected (Fig. 6D) network during excitation by randomly firing fibers (Fig $6 C, a, D, a$ ) or an equivalent constant current (Fig. $6 C, b, D, b$ ). Removing the noise improved synchrony, as assessed from the increase in CI from
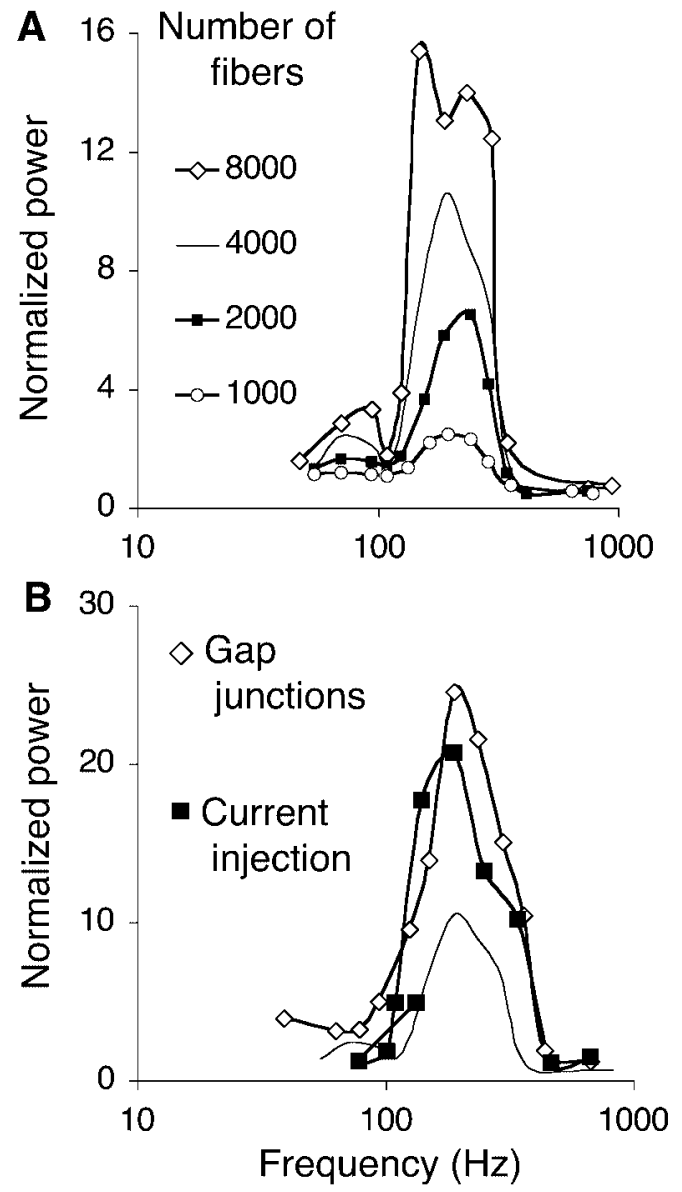

Figure 7. Noise, heterogeneity, and gap junctions modulate the power over more than an order of magnitude without affecting the resonance frequency. Tuning curves were calculated by simulating the one-dimensional network with only nearest neighbor coupling ( $d=1 \mathrm{msec}$; $\tau=3 \mathrm{msec}$ ); plain curves are the tuning curves from Figure $1 F$. All tuning curves yield a resonance frequency between 205 and $210 \mathrm{~Hz}$. A, Effect of varying the size of the population of afferent fibers (from 1000 to 8000). B, Tuning curve constructed by incrementing the intensity of a fixed constant current injected into the soma of each neuron (filled squares) and tuning curve obtained during fiber excitation after the addition of electrical synapses between mutually inhibitory neurons (open diamonds). Electrical synapses were pure conductors, with a conductance 0.4 times the unitary peak conductance of the chemical synapses between the same compartments.

0.178 (Fig. $6 C, a$ ) to 0.381 (Fig 6C, $b$ ) and from 0.237 (Fig 6D, $a$ ) to 0.465 (Fig. $6 D, b)$.

The residual asynchrony of the fully connected network (Fig. $6 D, b)$ must be attributed to the heterogeneity of neurons and synapses. The homogeneous network produced a CI of 0.98 (data not shown). We quantified in an indirect way the present degree of heterogeneity by increasing the variability of current intensity among the neurons of the homogeneous network. Distributed currents drawn from a Gaussian distribution with a CV equal to 0.02 reduced the CI from 0.98 to 0.453 , a value comparable with the CI of 0.465 in the raster plot of Figure $6 D, b$.

The noise level can also be changed by varying the number of afferent fibers. Because the membrane potential has a lower variance when large numbers of afferents excite a neuron through weak synapses, disconnected neurons fire more regularly when the number of fibers increases, whereas smaller numbers of fibers, activating stronger synapses, synchronize the disconnected neurons more precisely (Maex and De Schutter, 1998a,b; Maex et al., 2000). We examined which effect predominates in the gener- 
ation of network oscillations and always observed that power increased with the size of the fiber population (Fig. 7A). In the limit of an infinite number of fibers, excitation by fibers is identical to the injection of a constant current. The tuning curve constructed by current injection did not differ in shape from that obtained with afferent fibers (Fig. $7 B$, filled squares; $f_{\mathrm{R}}=209 \mathrm{~Hz}$ ).

Varying the degree of heterogeneity altered the peak power over almost two orders of magnitude without affecting much $f_{\mathrm{R}}$, although there was a tendency for low-frequency oscillations to be more robust than high-frequency oscillations.

\section{Adding gap junctions stabilizes the oscillations but does not change $f_{\mathrm{R}}$}

Closely spaced inhibitory neurons form dendritic gap junctions [ $>60 \%$ of neuron pairs separated $<50 \mu \mathrm{m}$ (Galarreta and Hestrin, 1999; Gibson et al., 1999) and all reciprocally connected pairs (Galarreta and Hestrin, 2002)]. In accordance with previous modeling studies (Traub et al., 2001; Bartos et al., 2002), the power of the network oscillations increased in a non-frequency-selective manner when adjacent neurons were connected by purely resistive electrical synapses (Fig. 7B, open diamonds). Hence, although the electrotonically transmitted afferent spike conceals the initial phase of the IPSP (see Tamás et al., 2000), this apparent increase in delay of the IPSP onset does not decrease the resonance frequency of the network $\left(f_{\mathrm{R}}=210 \mathrm{~Hz}\right)$.

\section{Mechanism of synchronization and frequency tuning}

Pairs of reciprocally connected inhibitory neurons tend to fire synchronously if each neuron, on firing, resets in an appropriate way the firing cycle of the paired neuron. In the present model neuron, activation of an inhibitory synapse delayed the generation of a spike, and the resulting increase of the interspike interval was greater the later in the firing cycle the synapse was activated (Fig. $8 A$, phase-response curve in $B$ ). As a consequence, of a pair of neurons reciprocally connected through inhibitory synapses, the neuron firing earlier received inhibition later and postponed its next spike more (Fig. $8 C$ ). Repetition of this mechanism progressively leads to a more precise synchronization of successive pairs of spikes (Ernst et al., 1995).

More precisely, let two mutually inhibitory neurons, denoted by superscripts 1 and 2, fire regularly with the same period $T$ in a disconnected network. If the neurons generated their most recent spikes, indicated by subscript $\mathrm{i}$, at $t_{\mathrm{i}}{ }^{1}$ and $t_{\mathrm{i}}{ }^{2}=t_{\mathrm{i}}{ }^{1}+\Delta_{\mathrm{i}}$, with $\Delta_{\mathrm{i}}<d$, then they receive inhibition in a connected network at time:

$$
t_{\mathrm{i}}^{2}+d=t_{\mathrm{i}}^{1}+\Delta_{\mathrm{i}}+d \text { and } t_{\mathrm{i}}^{1}+d,
$$

i.e., with a phase equal to:

$$
\begin{aligned}
\varphi^{1}=\left(t_{\mathrm{i}}^{1}+\Delta_{\mathrm{i}}+d-t_{\mathrm{i}}^{1}\right) / T & =\left(d+\Delta_{\mathrm{i}}\right) / T \text { and } \\
\varphi^{2} & =\left(t_{\mathrm{i}}^{1}+d-t_{\mathrm{i}}^{2}\right) / T=\left(d-\Delta_{\mathrm{i}}\right) / T .
\end{aligned}
$$

If the phase-response curve has a linear form:

$$
\left(t_{\mathrm{i}+1}-t_{\mathrm{i}}-T\right) / T=b+a \varphi,
$$

then the neurons will generate their next spikes at time:

$$
\begin{aligned}
& t_{\mathrm{i}+1}^{1}=t_{1}^{1}+T+T\left(b+a\left(d+\Delta_{\mathrm{i}}\right) / T\right) \text { and } \\
& \qquad t_{\mathrm{i}+1}^{2}=t_{\mathrm{i}}^{2}+T+T\left(b+a\left(d-\Delta_{\mathrm{i}}\right) / T\right),
\end{aligned}
$$

giving:

$$
\Delta_{\mathrm{i}+1}=t_{\mathrm{i}+1}^{2}-t_{\mathrm{i}+1}^{1}=\Delta_{\mathrm{i}}(1-2 a) .
$$

The neurons synchronize asymptotically if $\left|\Delta_{\mathrm{i}+1}\right|<\left|\Delta_{\mathrm{i}}\right|$, which requires $a<1$ (assuming $a>0$ ). Figure $8 C$ depicts the sequence of events for phase-response curves with slope $a<0.5$. For $0.5<$
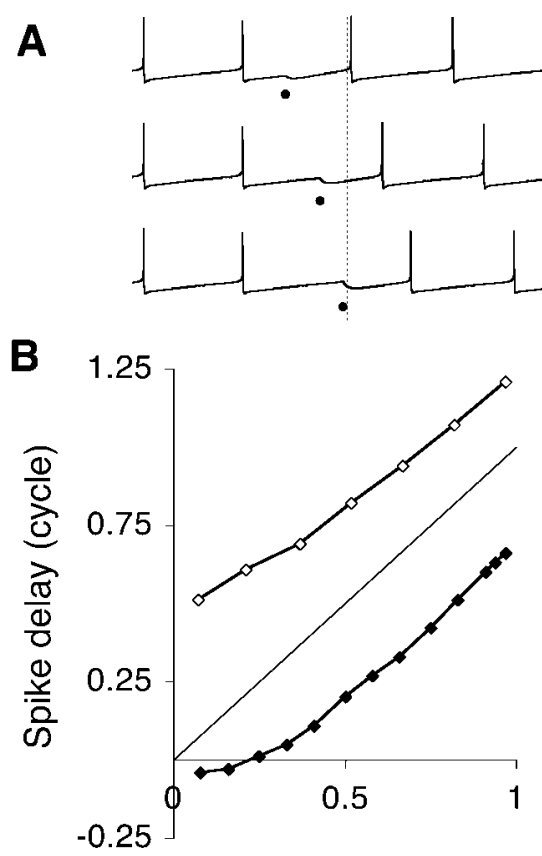

Timing of inhibition (cycle)
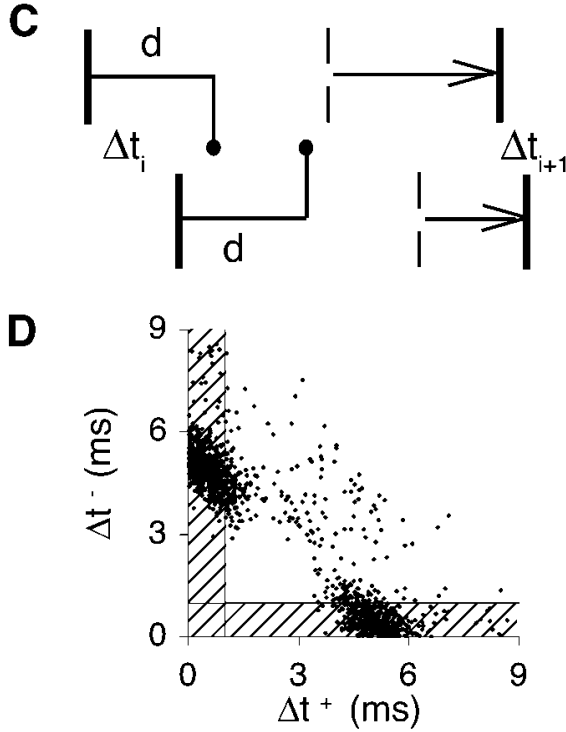

Figure 8. Mechanism of synchronization and frequency tuning. A, Activation of an inhibitory synapse resets the firing cycle of the model neuron. The synapse is activated after 15 (top membrane potential trace), 27 (middle trace), and 35 (bottom trace) msec in a $36 \mathrm{msec}$ firing cycle. Dots indicate times of synaptic activation, and the vertical dashed line indicates the end of the cycle in the absence of inhibition. $B$, Phase-response curve, plotting the delay in spike generation after the activation of an inhibitory synapse at varying time instants (Rinzel and Ermentrout, 1999). The $x$ - and $y$-axis variables are expressed as fractions of the duration of the firing cycle. The strength of the activated synapse was 1 or $3 \mathrm{mS} / \mathrm{cm}^{2}$ (filled, open symbols, respectively). A phase-resetting curve parallel to the diagonal line corresponds to a fixed delay elapsing between the activation of the inhibitory synapse and the generation of the next spike. C, Heuristic diagram of the synchronization mechanism. In one cycle, the interval between a pair of spikes from mutually inhibitory neurons (vertical bars) decreases from $\Delta t_{\mathrm{i}}<d$ to $\Delta t_{\mathrm{i}+1}$ (see Results). The broken vertical bars represent the expected spike times in the absence of inhibition. $D$, Relative timing of spikes from two adjacent neurons in the simulated network of Figure 1 ( $d=1 \mathrm{msec}$ ). Each spike of the reference neuron is represented as a dot, positioned according to the absolute time interval made with the previous $\left(\Delta \mathrm{t}^{+} ; x\right.$-axis) and next $\left(\Delta \mathrm{t}^{-} ; y\right.$-axis) spikes of a neighbor neuron. Most spikes led or lagged the neighbor neuron by an interval less than the delay $d$ of the synaptic response (shaded areas). 
$a<1.0$, the paired neurons still converge to synchrony, but the order of their spikes reverses at each cycle.

The frequency tuning of the network oscillations and, more particularly, the $1 /(4 d)$ frequency observed for resonance can be understood from the effect of inhibition on the instantaneous firing probability of a neuron. When the first neuron of a pair has fired, the second neuron lowers its firing rate after the onset of inhibition, i.e., after an interval equal to the delay $d$. Alternatively, the second neuron may have fired first, preceding the first neuron within an interval of the same duration, so that the second neuron will preferentially fire in an interval $[-d, d]$ around each spike of the first neuron (Fig. $8 D$ ). The sine wave best covering this $2 d$ window of increased firing has a $4 d$ period, and resonance occurs when both neurons, driven by the appropriate amount of excitatory input, fire on average with $4 d$ intervals. In addition, for $4 d$ to be the optimal period, inhibition must ensure that the $2 d$ windows of increased firing alternate with $2 d$ intervals of suppression of firing. This effect of the duration of inhibition explains the existence of an optimal decay time constant for each delay in Figure $3 A$.

\section{Discussion}

Delayed reciprocal inhibition is able to synchronize, with zero phase lag, homogeneous networks of pulse-coupled oscillators and was proposed to be a mechanism of neuronal synchronization (van Vreeswijk et al., 1994; Ernst et al., 1995). Simulating heterogeneous hippocampal interneuron networks, Bartos et al. (2002) concluded that "a rapid inhibitory signal generated with a certain delay is a very effective synchronization signal." The present study demonstrates that the resulting oscillations in network activity are limited to a narrow frequency band constrained by the IPSC latency but rather insensitive to the IPSC strength and decay time constant. Small, realistic axonal and synaptic delays, considered negligible compared with the low-pass time constant of the neuronal membrane (Manor et al., 1991), have a dramatic effect on the frequency spectrum (Fig. 1). The induced synchrony may be less precise than the synchrony that was achieved without explicit delays in more homogeneous networks (Wang and Buzsáki, 1996), but the robustness of the oscillations, their sharp tuning and frequency constancy, and their emergence in sparsely connected networks favor a model of delay-induced synchrony for fast brain rhythms. The extreme frequency control in these networks can be appreciated from the fact that variations in network heterogeneity did not affect the resonance frequency even when power changed by almost two orders of magnitude.

Oscillations obeying a $1 /(4 d)$ rule are observed in many biological systems (May, 1976; Glass and Mackey, 1988; MacDonald, 1989). In previous analytical models of oscillatory behavior in populations of neurons, delays attributable to signal propagation along axons and dendrites were lumped into the time constants of linear or nonlinear differential equations (Wilson and Cowan, 1972; Freeman, 1975). However, the incorporation of discrete delay variables can profoundly alter the dynamics of a system (MacDonald, 1989). Linear first-order delay systems can oscillate with a $4 d$ period, and nonlinear delay differential equations can exhibit, at a critical delay value, a bifurcation from a steady state to a limit cycle solution with a period approximately equal to four times the delay period. In Appendix, we derive a formal explanation for our present findings.

\section{Sources of delays in neural networks}

The present results hold irrespective of the source of the delay between the timing of the action potential of an inhibitory neu- ron and the onset of the current response in the paired postsynaptic neuron. Because the range of the delays that neural circuits are able to produce constrains the frequency range of the oscillations to which the present model may apply, we briefly discuss some prevalent mechanisms of delayed inhibition.

Although inhibitory neurons are fast-processing units, with an axon tree often confined within $600 \mu \mathrm{m}$ from the soma (Buhl et al., 1994; Sik et al., 1995), some (partly myelinated) axons can extend several millimeters in hippocampus (Sik et al., 1994) and neocortex (Kisvarday et al., 1993; Gupta et al., 2000). Salin and Prince (1996) electrically evoked monosynaptic IPSCs with latencies of 1 to $>6 \mathrm{msec}$ in neocortical pyramidal cells in vitro and derived slow speeds for spike propagation $(0.06-0.2 \mathrm{~m} / \mathrm{sec}$; mean, $0.1 \mathrm{~m} / \mathrm{sec}$ ). From our simulation data, a mean latency of 4 msec induces resonance at $62.5 \mathrm{~Hz}$ (Fig. 3), which is within the frequency range of the oscillations recorded in visual (Gray and Viana Di Prisco, 1997) and auditory neocortex (Brosch et al., 2002).

The reported latencies of unitary IPSCs, evoked by firing an impaled presynaptic neuron, are usually much shorter (mean, 0.8 msec for pairs $<200 \mu \mathrm{m}$ apart in dentate gyrus; Bartos et al., 2001), but paired recordings may be biased to small interelectrode distances at which the probability of finding connected neurons is highest. IPSP latencies of 3.2-8.6 msec (mean, 5.4 msec) were measured for pairs 153-445 $\mu \mathrm{m}$ apart in striatum (Tunstall et al., 2002), and in hippocampus, unitary IPSCs between lacunosum-moleculare interneurons and pyramidal cells showed latencies, albeit at room temperature, of $2.4-7.2 \mathrm{msec}$ (mean, 4.2 msec; Bertrand and Lacaille, 2001).

In addition, it is the mean distance to the entire set of postsynaptic neurons that determines the resonance frequency. This mean distance can be estimated from the experimentally derived connection probability function, provided the probability at each distance is corrected for the varying numbers of neurons available. For axons with a disk- or sphere-like arborization, the number of candidate target neurons increases linearly or quadratically with distance from the source neuron. Hence, if the connection probability is observed to fall off according to an exponential function with space constant $\delta$, the actual mean distance increases from $\delta$ to $2 \delta$ and $3 \delta$ for axons branching in two and three dimensions, respectively.

The present mechanism is not restricted to purely inhibitory networks. The $1 /(4 d)$ rule generalizes to circuits with intercalated excitatory neurons (for example $I_{1} \rightarrow E_{1} \rightarrow I_{2} \rightarrow E_{2} \rightarrow I_{1}$ instead of $I_{1} \rightarrow I_{2} \rightarrow I_{1}$ ). Here resonance is predicted to arise at an oscillation period equal to four times the delay of the disynaptic response $I_{1} \rightarrow E_{1} \rightarrow I_{2}$. If the response delay is much greater for excitatory than for inhibitory connections, then the circuit will resonate with a period approximately four times the delay of excitation, and, consequently, the excitatory neurons will lead the inhibitory neurons by one-fourth of a cycle. Such a phase relationship has been observed in olfactory systems (Freeman, 1975; Bazhenov et al., 2001) and hippocampus (Csicsvari et al., 2003).

Finally, circuits with excitatory feedback can generate trains of spikes with delays of tens to hundreds of milliseconds. In visual cortex, feedback excitation was proposed to provide the long delays of inhibition needed to generate directionally selective responses to slowly moving stimuli (down to $<1 \mathrm{~Hz}$; Maex and Orban, 1996). It is noteworthy in this respect that the property of directional selectivity, which can be implemented in a circuit with lateral inhibition, exhibits the same $f=1 /(4 d)$ relationship, $f$ being the temporal frequency of the moving stimulus evoking maximally selective responses (van Santen and Sperling, 1985). 


\section{Predictions}

The present results and the above arguments lead to the following predictions, some of which are substantiated by recent findings. The delay of monosynaptic or polysynaptic inhibition is the critical parameter determining the resonance frequency of an inhibitory network. Hence, oscillations with different characteristic frequencies are expected to be generated by microcircuits involving different types of interneurons (Klausberger et al., 2003). In these interneurons and in their target projection neurons, IPSCs are predicted to be evoked with variable latencies, centered at approximately one-fourth of the oscillation period (Traub et al., 1996). Because the multitude of microcircuits in the brain could generate a continuum of delays, probably with slight differences emerging between different areas or developmental stages, oscillations in the nervous system could form a continuum rather than being divided into a few discrete frequency bands (Csicsvari et al., 1999; Grenier et al., 2001). Interneurons involved in circuits with low characteristic frequencies are predicted to fire multispike bursts during each cycle. Finally, although the delay of reciprocal inhibition might not be very amenable to experimental manipulation, changing the size of the stimulus would vary the effective mean delay. Stimuli exciting a focus smaller than the connection radius of an inhibitory network do not recruit longdistance neuron pairs, leading to faster oscillations than predicted from the mean delay of the circuit (Bartos et al., 2002, their Fig. $4 E$ ). Fast synchronous oscillations are therefore predicted to be more narrowly localized than low-frequency oscillations, although long-distance excitatory connections may contribute to this difference (Ermentrout and Kopell, 1998; Pauluis et al., 1999; Kopell et al., 2000).

\section{Conclusions}

We propose that resonant synchronization induced by the delay of (monosynaptic or polysynaptic) inhibitory connections contributes to the emergence and frequency tuning of all types of oscillations in which inhibitory synapses are involved, and that delay-induced synchronization should be considered for fast oscillations in particular $(>40 \mathrm{~Hz})$. Some types of fast oscillations appear to persist, however, in the absence of synaptic transmission (200 Hz "ripples"), and axoaxonal gap junctions may be essential for their generation (Schmitz et al., 2001). For other types of oscillations, additional tuning mechanisms may be important, such as intrinsic neuronal resonance and synaptic dynamics (Gupta et al., 2000; Beierlein et al., 2003), which were not included in the present model.

\section{Appendix}

Let $A(t)$ be the population activity at time $t, F$ the neuronal transfer function, $I$ the constant level of external input, $K(t)$ the synaptic impulse response, and $d$ the combined axonal and synaptic delay. Then the dynamics of a fully connected, homogeneous network can be described by:

$$
\lambda d A / d t+A(t)=F(I-K \cdot A(t-d)) .
$$

where $\cdot$ denotes convolution in time (Gerstner, 2000). During asynchronous firing, the population activity is considered constant over time: $A(t)=C=F(I-C)$. Linearization about the asynchronous state yields:

$$
\begin{aligned}
\lambda d A / d t+A(t)=F( & -C+C-K \cdot A(t-d)) \approx F(I-C) \\
& +F^{\prime}(I-C)(C-K \cdot A(t-d)) . \quad(\mathrm{A} 2)
\end{aligned}
$$

The linearized equation, after substituting $y(t)$ for $A(t)-F(I-$ $C)$ and $a$ for the derivative of $F$ is:

$$
\lambda d y / d t+y(t)=-a K \cdot y(t-d) .
$$

We take $K(t)=(1 / \tau) \exp (-t / \tau)$ and follow the reasoning developed by MacDonald (1989), looking for a periodic solution $y=$ $\exp (i \omega t)$, with $\omega=2 \pi / T$. We consider two cases. The first case explains the observation in Figure $3 B$ that for networks with low resonance frequency, $f_{\mathrm{R}}$ is slightly $>1 /(4 d)$ but almost completely independent of the decay time constant $\tau$. The second case matches the observation that networks with a high resonance frequency can have an $f_{\mathrm{R}}$ of $\leq 1 /(4 d)$, which in addition is more dependent on the value of $\tau$.

At low resonance frequencies (case 1), the synaptic kinetics is much faster than both the membrane time constant $\lambda$ and the oscillation period $T$, so that the delayed feedback can be considered to be approximately instantaneous. This reduces Equation A3 to:

$$
\lambda d y / d t+y(t)=-a y(t-d)
$$

The resulting transcendental characteristic equation:

$$
i \omega \lambda+1=-a e^{-i \omega \mathrm{d}}
$$

has real roots for $\cos (\omega d)=-1 / a$ and $\sin (\omega d)=\omega \lambda / a$, or:

$$
\tan (\omega d)=-(\omega d)(\lambda / d)
$$

Provided $a>1$, solving for $\omega d$ yields $\pi / 2<\omega d<\pi$, giving $2 d<$ $T<4 d$. Hence, $f_{\mathrm{R}}>1 /(4 d)$ but independent of $\tau$.

At high resonance frequencies and hence high levels of excitation (case 2), the membrane potential of a neuron is strongly depolarized, and voltage deflections are small (although sufficient to pass the firing threshold) because of the low-pass characteristics of the membrane. Hence the contribution of the leak current to membrane dynamics can, approximately, be ignored. Equation A3 now reduces to:

$$
\lambda d y / d t=-a K \cdot y(t-d) .
$$

The resulting transcendental characteristic equation is:

$$
i \omega \lambda(1+i \omega \tau)=-a e^{-\mathrm{i} \omega \mathrm{d}} .
$$

For very fast synapses in the limit $\tau \rightarrow 0$, the solution is $\omega d=\pi / 2$, or $T=4 d$. For $\tau>0$, the present case is an instance of a distributed delay (the synaptic kernel) with a gap (the synaptic delay $d$ ), and $T>4 d$ with $T$ increasing for increasing $\tau$ (MacDonald, 1989).

Note added in proof. It was brought to our attention that Brunel and Wang (2003) reported similar results recently.

\section{References}

Baçar E (1998) Brain function and oscillations. Berlin: Springer.

Bartos M, Vida I, Frotscher M, Geiger JRP, Jonas P (2001) Rapid signaling at inhibitory synapses in a dentate gyrus interneuron network. J Neurosci 21:2687-2698.

Bartos M, Vida I, Frotscher M, Meyer M, Monyer H, Geiger JRP, Jonas P (2002) Fast synaptic inhibition promotes synchronized gamma oscilla- 
tions in hippocampal interneuron networks. Proc Natl Acad Sci USA 99:13222-13227.

Bazhenov M, Stopfer M, Rabinovich M, Huerta R, Abarbanel HDI, Sejnowski TJ, Laurent G (2001) Model of transient oscillatory synchronization in the locust antennal lobe. Neuron 30:553-567.

Beierlein M, Gibson JR, Connors BW (2003) Two dynamically distinct inhibitory networks in layer 4 of the neocortex. J Neurophysiol, in press.

Bertrand S, Lacaille JC (2001) Unitary synaptic currents between lacunosum-moleculare interneurones and pyramidal cells in rat hippocampus. J Physiol (Lond) 532:369-384.

Bragin A, Jando G, Nadasdy Z, Hetke J, Wise K, Buzsáki G (1995) Gamma $(40-100 \mathrm{~Hz})$ oscillation in the hippocampus of the behaving rat. J Neurosci 15:47-60.

Brosch M, Budinger E, Scheich H (2002) Stimulus-related gamma oscillations in primate auditory cortex. J Neurophysiol 87:2715-2725.

Brunel N, Wang X-J (2003) What determines the frequency of fast network oscillations with irregular neural discharges? I. Synaptic dynamics and excitation-inhibition balance. J Neurophysiol 90:415-430.

Buhl EH, Halasy K, Somogyi P (1994) Diverse sources of hippocampal unitary inhibitory postsynaptic potentials and the number of synaptic release sites. Nature 368:823-828.

Bush P, Sejnowski T (1996) Inhibition synchronizes sparsely connected cortical neurons within and between columns in realistic network models. J Comput Neurosci 3:91-110.

Buzsáki G, Chrobak JJ (1995) Temporal structure in spatially organized neuronal ensembles: a role for interneuronal networks. Curr Opin Neurobiol 5:504-510.

Carter AG, Regehr WG (2002) Quantal events shape cerebellar interneuron firing. Nat Neurosci 5:1309-1318.

Cheron G, Gall D, Meyer M, Schurmans S, Schiffmann S (2001) Highfrequency oscillation in the cerebellum of the double calretinin-calbindin knockout mice. Soc Neurosci Abstr 27:294.10.

Cobb SR, Halasy K, Vida I, Nyiri G, Tamás G, Buhl EH, Somogyi P (1997) Synaptic effects of identified interneurons innervating both interneurons and pyramidal cells in the rat hippocampus. Neuroscience 79:629-648.

Csicsvari J, Hirase H, Czurkó A, Mamiya A, Buzsáki G (1999) Fast network oscillations in the hippocampal CA1 region of the behaving rat. J Neurosci 19:RC20(1-4).

Csicsvari J, Jamieson B, Wise KD, Buzsáki G (2003) Mechanism of gamma oscillations in the hippocampus of the behaving rat. Neuron 37:311-322.

Ermentrout GB, Kopell N (1998) Fine structure of neural spiking and synchronization in the presence of conduction delays. Proc Natl Acad Sci USA 95:1259-1264.

Ernst U, Pawelzik K, Geisel T (1995) Synchronization induced by temporal delays in pulse-coupled oscillators. Phys Rev Lett 74:1570-1573.

Farmer SF (1998) Rhythmicity, synchronization and binding in human and primate motor systems. J Physiol (Lond) 509:3-14.

Freeman WJ (1975) Mass action in the nervous system. New York: Academic.

Galarreta M, Hestrin S (1999) A network of fast-spiking cells in the neocortex connected by electrical synapses. Nature 402:72-75.

Galarreta M, Hestrin S (2002) Electrical and chemical synapses among parvalbumin fast-spiking GABAergic interneurons in adult mouse neocortex. Proc Natl Acad Sci USA 99:12438-12443.

Gerstner W (2000) Population dynamics of spiking neurons: fast transients, asynchronous states, and locking. Neural Comput 12:43-89.

Gibson JR, Beierlein M, Connors BW (1999) Two networks of electrically coupled inhibitory neurons in neocortex. Nature 402:75-79.

Glass L, Mackey MC (1988) From clocks to chaos. Princeton, NJ: Princeton UP.

Gray CM (1994) Synchronous oscillations in neuronal systems: mechanisms and functions. J Comput Neurosci 1:11-38.

Gray CM, Viana Di Prisco G (1997) Stimulus-dependent neuronal oscillations and local synchronization in striate cortex of the alert cat. J Neurosci 17:3239-3253.

Grenier F, Timofeev I, Steriade M (2001) Focal synchronization of ripples $(80-200 \mathrm{~Hz})$ in neocortex and their neuronal correlates. J Neurophysiol 86:1884-1898.

Gupta A, Wang Y, Markram H (2000) Organizing principles for a diversity of GABAergic interneurons and synapses in the neocortex. Science 287: 273-278.
Hartline KK, Ratliff F (1972) Inhibitory interactions in the retina of Limulus. In: Handbook of sensory physiology, Vol VII/2, Physiology of photoreceptor organs (Fuortes MGF, ed), pp 381-447. Berlin: Springer.

Kandel A, Buzsáki G (1997) Cellular-synaptic generation of sleep spindles, spike-and-wave discharges, and evoked thalamocortical responses in the neocortex of the rat. J Neurosci 17:6783-6797.

Kisvarday ZF, Beaulieu C, Eysel UT (1993) Network of GABAergic large basket cells in cat visual cortex (area 18): implication for lateral disinhibition. J Comp Neurol 327:398-415.

Klausberger T, Magill PJ, Marton LF, Roberts JD, Cobden PM, Buzsáki G, Somogyi P (2003) Brain-state- and cell-type-specific firing of hippocampal interneurons in vivo. Nature 421:844-848.

Kopell N, Ermentrout GB, Whittington MA, Traub RD (2000) Gamma rhythms and beta rhythms have different synchronization properties. Proc Natl Acad Sci USA 97:1867-1872.

Liley DTJ, Cadush PJ, Dafilis MP (2002) A spatially continuous mean field theory of electrocortical activity. Network 13:67-113.

MacDonald N (1989) Biological delay systems: linear stability theory. Cambridge, UK: Cambridge UP.

Maex R, De Schutter E (1998a) Synchronization of Golgi and granule cell firing in a detailed network model of the cerebellar granule cell layer. J Neurophysiol 80:2521-2537.

Maex R, De Schutter E (1998b) The critical synaptic number for rhythmogenesis and synchronization in a network model of the cerebellar granular layer. In: Proceedings of the Eighth International Conference on Artificial Neural Networks (Skovde, Sweden) (Niklasson L, Boden M, Ziemke T, eds), pp 361-366. London: Springer.

Maex R, Orban GA (1996) Model circuit of spiking neurons generating directional selectivity in simple cells. J Neurophysiol 75:1515-1545.

Maex R, Vos BP, De Schutter E (2000) Weak common parallel fibre synapses explain the loose synchrony observed between rat cerebellar Golgi cells. J Physiol (Lond) 523:175-192.

Maex RL, Solinas S, Cheron G, Schiffmann S, De Schutter E (2002) The plexus of recurrent Purkinje axon collaterals: a network for fast synchronization? Soc Neurosci Abstr 360.11

Manor Y, Koch C, Segev I (1991) Effect of geometrical irregularities on propagation delay in axonal trees. Biophys J 60:1424-1437.

May RM (1976) Theoretical biology. Oxford, UK: Blackwell.

McBain CJ, Fisahn A (2001) Interneurons unbound. Nat Revs Neurosci 2:11-23.

Pauluis Q, Baker SN, Olivier E (1999) Emergent oscillations in a realistic network: the role of inhibition and the effect of the spatiotemporal distribution of the input. J Comput Neurosci 6:27-48.

Press WH, Teukolsky SA, Vetterling WT, Flannery BP (1988) Numerical recipes in C. Cambridge, UK: Cambridge UP.

Rinzel J, Ermentrout B (1999) Analysis of neural excitability and oscillations. In: Methods in neuronal modeling: from ions to networks (Koch C, Segev I, eds), pp 249-291. Cambridge, MA: MIT.

Ropert N, Miles R, Korn H (1990) Characteristics of miniature inhibitory postsynaptic currents in CA1 pyramidal neurones of rat hippocampus. J Physiol (Lond) 428:707-722.

Salin PA, Prince DA (1996) Electrophysiological mapping of $\mathrm{GABA}_{\mathrm{A}}$ receptor-mediated inhibition in adult rat somatosensory cortex. J Neurophysiol 75:1589-1600.

Schmitz D, Schuchmann S, Fisahn A, Draguhn A, Buhl EH, Petrasch-Parwez E, Dermietzel R, Heinemann U, Traub RD (2001) Axo-axonal coupling: a novel mechanism for ultrafast neuronal communication. Neuron 31:831-840.

Sik A, Ylinen A, Penttonen M, Buzsáki G (1994) Inhibitory CA1-CA3-hilar region feedback in the hippocampus. Science 265:1722-1724.

Sik A, Penttonen M, Ylinen A, Buzsáki G (1995) Hippocampal CA1 interneurons: an in vivo intracellular labeling study. J Neurosci 15:6651-6665.

Tamás G, Somogyi P, Buhl EH (1998) Differentially interconnected networks of GABAergic interneurons in the visual cortex of the cat. J Neurosci 18:4255-4270.

Tamás G, Buhl EH, Lörincz A, Somogyi P (2000) Proximally targeted GABAergic synapses and gap junctions synchronize cortical interneurons. Nat Neurosci 3:366-371. 
Tiesinga PHE, José JV (2000) Robust gamma oscillations in networks of inhibitory hippocampal interneurons. Network 11:1-23.

Traub RD, Whittington MA, Colling SB, Buzsáki G, Jefferys JGR (1996) Analysis of gamma rhythms in the rat hippocampus in vitro and in vivo. J Physiol (Lond) 493:471-484.

Traub RD, Jefferys JGR, Whittington MA (1999) Fast oscillations in cortical circuits. Cambridge, MA: MIT.

Traub RD, Kopell N, Bibbig A, Buhl EH, LeBeau FEN, Whittington MA (2001) Gap junctions between interneuron dendrites can enhance synchrony of gamma oscillations in distributed networks. J Neurosci 21:9478-9486.

Tunstall MJ, Oorschot DE, Kean A, Wickens JR (2002) Inhibitory interactions between spiny projection neurons in the rat striatum. J Neurophysiol 88:1263-1269.

van Santen JPH, Sperling G (1985) Elaborated Reichardt detectors. J Opt Soc Am A Opt Image Sci Vis 2:300-321. van Vreeswijk C, Abbott LF, Ermentrout GB (1994) When inhibition not excitation synchronizes neural firing. J Comput Neurosci 1:313-321.

Wang X-J, Buzsáki G (1996) Gamma oscillations by synaptic inhibition in a hippocampal interneuronal network model. J Neurosci 16:6402-6413.

Wang X-J, Rinzel J (1993) Spindle rhythmicity in the reticularis thalami nucleus: synchronization among mutually inhibitory neurons. Neuroscience 53:899-904.

White JA, Chow CC, Ritt J, Soto-Treviño C, Kopell N (1998) Synchronization and oscillatory dynamics in heterogeneous, mutually inhibited neurons. J Comput Neurosci 5:5-16.

Whittington MA, Traub RD, Jefferys JGR (1995) Synchronized oscillations in interneuron networks driven by metabotropic glutamate receptor activation. Nature 373:612-615.

Wilson HR, Cowan JD (1972) Excitatory and inhibitory interactions in localized populations of model neurons. Biophys J 12:1-24. 